\title{
1 Genetic regulation of the bacterial omega-3 polyunsaturated \\ 2 fatty acid biosynthesis pathway
}

$4 \quad \operatorname{Marco}$ N. Allemann ${ }^{\text {a,b }}$ and Eric E. Allen ${ }^{\text {a,b,c }}$

6 aarine Biology Research Division, Scripps Institution of Oceanography, University of

7 California, San Diego, La Jolla, CA USA

$8 \quad{ }^{b}$ Center for Marine Biotechnology and Biomedicine, Scripps Institution of

9 Oceanography, University of California, San Diego, La Jolla, CA USA

$10{ }^{c}$ Division of Biological Sciences, University of California, San Diego, La Jolla, CA USA

$11 *$ *orresponding author: e-mail address: $\underline{\text { eallen@ucsd.edu }}$

13 Running Head: Identification of a novel fatty acid regulator

\section{Abstract}

16 A characteristic among many marine Gammaproteobacteria is the biosynthesis

17 and incorporation of omega-3 polyunsaturated fatty acids into membrane phospholipids.

18 Biosynthesis of eicosapentaenoic (EPA) and/or docosahexaenoic (DHA) acids is

19 accomplished using a polyketide/fatty acid synthase mechanism encoded by a set of five

20 genes $p f a A B C D E$. This unique fatty acid synthesis (FAS) pathway co-exists with the

21 canonical Type II dissociated fatty acid synthesis pathway, which is responsible for the

22 biosynthesis of saturated, monounsaturated, and hydroxylated fatty acids used in

23 phospholipid and lipid A biosynthesis. In this work, a genetic approach was undertaken

24 to elucidate genetic regulation of the $p f a$ genes in the model marine bacterium

25 Photobacterium profundum SS9. Using a reporter gene fusion, we showed that 
26 expression of the $p f a$ operon is down regulated in response to exogenous fatty acids,

27 particularly long chain monounsaturated fatty acids. This regulation occurs independently

28 of the canonical fatty acid regulators, FabR and FadR, present in P. profundum SS9.

29 Transposon mutagenesis and screening of a library of mutants identified a novel

30 transcriptional regulator, which we have designated $p f a F$, to be responsible for the

31 observed regulation of the $p f a$ operon in P. profundum SS9. Gel mobility shift and DNase

32 I footprinting assays confirmed that PfaF binds the $p f a A$ promoter and identified the PfaF

33 binding site.

\section{Importance}

36 The production of polyunsaturated fatty acids (PUFA) by marine Gammaproteobacteria,

37 particularly those from deep-sea environments, has been known for decades. These

38 unique fatty acids are produced by a polyketide-type mechanism and subsequently

39 incorporated into the phospholipid membrane. While much research has focused on the

40 biosynthesis genes, their products and the phylogenetic distribution of these gene

41 clusters, no prior studies have detailed the genetic regulation of this pathway. This study

42 describes how this pathway is regulated under various culture conditions and has

43 identified and characterized a fatty acid responsive transcriptional regulator specific to

44 the PUFA biosynthesis pathway.

\section{Introduction}

47 Regulation of fatty acid biosynthesis, particularly the levels of unsaturated fatty

48 acids, has been shown to be a crucial aspect of the bacterial physiological response to a 
49 variety of environmental conditions, including temperature, $\mathrm{pH}$, and hydrostatic pressure.

50 Both biochemical and transcriptional regulatory mechanisms exist to regulate the various

51 aspects of the fatty acid biosynthetic pathway (1). In the model organism Escherichia

52 coli, genes that compromise the Type II fatty acid synthase (FAS), and in particular genes

53 related to monounsaturated fatty acid (MUFA) biosynthesis are regulated by the interplay

54 between FadR and FabR (2-4) as shown in Figure 1. FadR is a member of the GntR

55 regulator family and it acts as positive regulator of both $f a b A$ and $f a b B$, which encode for

56 proteins essential to the biosynthesis of unsaturated fatty acids in this organism (5). As

57 seen in Figure 1 exogenous fatty acids $(>\mathrm{C} 10)$ are imported across the outer membrane

58 via the FadL transporter and subsequently converted into acyl-CoAs by the acyl-CoA

59 synthetase FadD. In the absence of long chain acyl-CoA, FadR binds to sites upstream of

$60 f a b A$ and $f a b B$ and acts as a positive regulator $(6,7)$. Upon acyl-CoA binding to FadR, it

61 adopts a conformation that is unable to bind to its cognate sites upstream of $f a b A / B$

62 promoters leading to deactivation of transcription. FabR, a TetR family transcriptional

63 regulator, further regulates $f a b A / B$ by acting as a classical repressor. FabR binds to sites

64 immediately downstream of the FadR site in both $f a b A / B$ regardless of acyl-CoA being

65 present $(3,8)$. While an exact role of FabR has yet to be described, it has been speculated

66 that the opposing actions of FabR and FadR at $f a b A$ and $f a b B$ promoters is ultimately

67 responsible for the regulation of these genes (3).

68 Similar regulatory mechanisms regulating monounsaturated fatty acid

69 biosynthesis have been characterized in other model Gram-negative bacterial systems. In

70 Shewanella oneidensis MR-1 the FabR homolog was found to be responsible for the

71 regulation of $f a b A$ and $\operatorname{des} A$, an oxygen dependent membrane bound lipid desaturase (9). 
72 Similar regulatory mechanisms controlling unsaturated lipid biosynthesis have been

73 described in other model bacteria such as Pseudomonas aeruginosa PAO1, which lacks a

$74 f a d R$ homolog $(1,10,11)$. In this strain $f a b A$ and $f a b B$ form an operon (unlike E. coli),

75 which is regulated by another TetR family regulator, DesT (12). In addition to fabAB

76 expression, DesT also modulates the expression of the membrane bound desaturase

77 DesBC, that catalyzes oxygen dependent desaturation of saturated acyl-CoA which can

78 then be incorporated into membrane phospholipids $(10,11)$.

79 A subset of marine Gammaproteobacteria, particularly strains isolated from cold

80 and/or high-pressure environments, produce omega-3 polyunsaturated fatty acids (PUFA)

81 such as EPA (20:5n-3) and DHA (22:6n-3), that are incorporated into phospholipid

82 membranes $(13,14)$. The biosynthesis of these unique fatty acids is linked to the

$83 p f a A B C D E$ operon, which encodes for a type I FAS/polyketide synthase (15). In these

84 bacteria, the Pfa synthase pathway co-exists with the Type II FAS, which produces

85 saturated (SFA) and monounsaturated fatty acids $(14,16)$. Given that both pathways

86 utilize the same precursor substrates $(15,17,18)$ and their respective end products are

87 destined for phospholipids $(13,14,19-22)$, an interesting question arises as to how these

88 pathways are physiologically coordinated in the cell. Numerous studies have

89 demonstrated that culturing native PUFA-producing strains at cold temperature (23-28)

90 and/or high pressure $(23,26)$ leads to increases in PUFA abundance. In the EPA

91 producing bacterium Photobacterium profundum SS9, analyses of transcript abundances

92 of the $p f a$ operon at cold temperatures and/or high pressure indicated no significant

93 alterations mRNA abundances relative to $15^{\circ} \mathrm{C}$ or low-pressure conditions (25). In the

94 course of those studies, a chemical mutant of P. profundum SS9 (EA2), was shown to 
95 have increased mRNA abundance relative to its parental strain indicating the possibility

96 of a transcriptional regulator(s) existing in the strain (25).

97 In this work, the transcriptional regulation of the $p f a$ operon in P. profundum SS9

98 is further characterized and shown that it is down regulated in response to exogenous

99 unsaturated fatty acid (18:1) supplementation. A genetic screen utilizing a $p f a A:: l a c Z Y$

100 reporter gene fusion combined with transposon mutagenesis was used to identify a novel

101 transcriptional regulator, herein designated $p f a F$, which positively regulates the $p f a$

102 operon and mediates the regulatory response to exogenous fatty acids. Gel mobility shift

103 and assays using purified PfaF demonstrated that PfaF binds to the $p f a A$ promoter and

104 that this binding is sensitive to the presence of oleoyl-CoA. DNase I footprinting was

105 used to determine the binding site of PfaF within the $p f a A$ promoter.

\section{Results}

\section{Expression of the pfa operon under various culture conditions}

109 To better understand the regulation of the $p f a$ operon and to facilitate monitoring

110 of gene expression, a reporter construct was designed to link expression of the $p f a$ operon

111 to the $l a c Z Y$ operon of Escherichia coli. This $p f a A:: l a c Z Y$ strain allows the $p f a$ operon

112 promoter to be monitored in single copy with all possible upstream regulatory sequences.

113 As expected from previous work (25) in P. profundum SS9, loss of $p f a A$ rendered the

$114 p f a A:: l a c Z Y$ strain unable to produce EPA (data not shown). Assays for $\beta$-galactosidase

115 activity indicated that in mid-log phase growth approximately 48 Miller units of activity

116 were produced (Figure 2A). Further $\beta$-galactosidase assays under conditions shown

117 previously to lead to increased EPA content, such as high hydrostatic pressure and low 
118 temperature are given in Figure 2A and indicated no changes in LacZ activity, confirming

119 previous results from this strain (25). Sequence analysis of the promoter region from

120 EA2, a previously isolated EPA overproducing strain with increased $p f a$ operon transcript

121 levels, also indicated no changes in the promoter sequence of strain EA2 relative to the 122 wild-type.

123 Given its biosynthetic role in producing fatty acids destined for phospholipid

124 biosynthesis, we hypothesized that the $p f a$ operon might be regulated in a similar fashion

125 to the prototypical fab regulon. Given the previously noted solubility issues of fatty acids

126 in 2216 marine growth medium (23), exogenous fatty acids in the form of various

127 polysorbate esters (Tween $20(12: 0), 40(16: 0), 60(18: 0), 80(18: 1))$ were utilized as

128 exogenous fatty acid supplements. As shown in Figure $2 \mathrm{~B}$, significant decreases in $\beta$ -

129 galactosidase activity were observed in the $p f a A:: l a c Z Y$ strain in response to all Tween

130 compounds except for Tween 20 (12:0) (Figure 2B). Given the various degrees of down-

131 regulation noted amongst the various Tween compounds seen in Figure 2B, which differ

132 only in their fatty acid component, the possibility of this response being due to the

133 polysorbate component of these compounds can be eliminated. This down-regulation was

134 also shown to occur in SS9R as both $p f a A$ and $p f a D$ transcript abundances are reduced

$135 \sim 3$-fold and $\sim 2$-fold, respectively, in response to Tween 80 (18:1) supplementation

136 (Figure 2C). Transcript abundances of $f a b A$ and $f a b B$ were also decreased under these

137 conditions (Figure 3C) indicating that, similar to the case in E. coli $(2-4,8)$, the

138 monounsaturated fatty acid (MUFA) biosynthesis genes are down regulated in the

139 presence of exogenous Tween 80 (18:1). Fatty acid profiling of SS9R grown in the

140 presence of Tween 80 supplementation indicated a nearly 4-fold increase in 18:1 
141 composition and an approximate 10-fold decrease in EPA consistent with the down-

142 regulation of $p f a A$ noted above (Supplemental Table 2). A novel fatty acid, tentatively

143 identified as 18:2, was also observed in cultures supplemented with Tween 80 and may

144 be representative of further metabolic processing of the incoming 18:1 acyl chain.

145 Role of FabR/FadR in response to exogenous fatty acids

146 Given the strong exogenous fatty acid phenotype, it was suspected that either

147 FadR and/or FabR, which are known to regulate E. coli fabA and $f a b B$ in response to

148 exogenous fatty acids, may be responsible for this regulatory phenomenon. Homologs of

149 fabR (locus tag: PBPRA3467) and fadR (locus tag: PBPRA2608) were readily identified

150 in the SS9 genome via homology searches and deletion mutants of both genes were

151 generated in both SS9R and $p f a A:: l a c Z Y$ strain backgrounds (Table 1). As shown in

152 Figure $3 \mathrm{~A}$, strains containing the $\Delta f a d R$ mutation displayed decreased $\beta$-galactosidase

153 activities. Comparing $\beta$-galactosidase activities of the $p f a A: \because l a c Z Y$ reporter strains

154 containing either $\Delta f a d R$ or $\Delta f a b R \Delta f a d R$ deletions indicated that both strains have similar

$155 \beta$-galactosidase activities and further ruled out the involvement of FabR in the regulation

156 of the $p f a$ operon. Interestingly, $\beta$-galactosidase assays of the $\Delta f a b R \Delta f a d R$ mutant

157 indicated that the down regulation of the $p f a A:: l a c Z Y$ gene fusion in the presence of

158 Tween 80 (18:1) was independent of both FadR and FabR (Figure 3A). Transcript

159 abundance analyses performed on RNA samples from the $\Delta f a b R \Delta f a d R$ mutant grown in

160 the presence or absence of Tween $80(18: 1)$ indicated that both $p f a A$ and $p f a D$ transcripts

161 were down regulated in response to supplementation while $f a b A$ and $f a b B$ transcripts

162 were essentially equivalent between the two conditions (Figure 3B). Fatty acid analyses

163 of the corresponding single and double mutant derivatives of SS9R are shown in 
164 Supplemental Table 2 and indicate that strains containing the $\Delta$ fadR mutation had

165 decreased EPA levels consistent with the reduced $\beta$-galactosidase activities noted. As

166 predicted, given the role of FadR as a positive regulator of $f a b A / B$, a decrease in

167 monounsaturated fatty acids was noted in strains containing the $\Delta f a d R$ mutation

168 (Supplemental Table 2).

169 Identification of a regulator specific to the pfa operon

170 The FadR/FabR independent down regulation of the $p f a$ operon in response to

171 Tween 80 (18:1) observed here suggested that another regulator(s) for the $p f a$ operon

172 might exist in $P$. profundum SS9. To search for additional regulators, the $p f a A:: l a c Z Y$

173 strain was subjected to transposon mutagenesis using the mini- $\operatorname{Tn} 5$ delivery vector

174 pRL27(29, 30). Of the approximately 10,000 mutants screened, several LacZ down or

175 loss of LacZ activity mutants were identified and saved for further analysis. Arbitrary

176 PCR was performed to identify the sites of mini-Tn5 insertion in these mutants.

177 Excluding mutants that had Tn5 insertions in the reporter gene and/or fadR, we identified

178 mutants from independent libraries that contained unique transposon insertions in the

179 same gene PBPRA0221 (TetR bacterial transcriptional regulator, pfam13972) (Figure

180 4A). One of these mutants displayed a five-fold decrease in LacZ activity and no longer

181 responded to exogenous Tween 80 (18:1) supplementation (Figure 4B). The PBPRA0221

182 gene, herein designated $p f a F$, encodes for a protein that is a member of the TetR family

183 of transcriptional regulators and is clustered with genes related to lipopolysaccharide

184 synthesis. To verify that this locus is involved in $p f a$ gene regulation, single crossover

185 insertion mutants were generated in the parental $p f a A:: l a c Z Y$ reporter strain and the EPA-

186 producing SS9R strain. The LacZ activity of the resulting strain was identical to that seen 
187 in the $p f a F:: \operatorname{Tn} 5$ mutant thereby verifying this relationship and excluding the possibility

188 of additional transposon insertion events being responsible for the observed phenotype

189 (data not shown). Transcript abundance analysis of the $p f a F$ mutant strain indicated

190 significant down-regulation of both $p f a A$ and $p f a D$ transcripts relative to SS9R (Figure

1914 C) consistent with the LacZ activity data. Additionally, quantification of $f a b A$ and $f a b B$

192 transcripts indicated no major differences between SS9R and its $p f a F$ mutant derivative.

193 A comparison of the fatty acid profiles of SS9R and a $p f a F$ mutant grown at $15^{\circ} \mathrm{C}$ shows

194 the mutant displays an approximate 4-fold reduction in EPA content relative to the wild-

195 type (Figure 3). The minor difference in the abundances of other fatty acids in the $p f a F$

196 mutant is also consistent with the minimal changes in $f a b A$ or $f a b B$ transcription. In the

197 case of both SS9R $\left(\mathrm{EPA}^{+}\right)$and $p f a A:: \operatorname{lacZY}\left(\mathrm{EPA}^{-}\right)$strains, genetic disruption of $p f a F$ did

198 not lead to alteration in growth capabilities at high pressure or cold temperatures (data not

199 shown).

200 The $p f a F$ gene was cloned onto the broad host range complementation plasmid

201 pFL122 and the resulting construct (pMA62) was introduced into $p f a F::$ Tn5 and SS9R

$202 p f a F$ mutant strains. As shown in Figure 4B, expression of $p f a F$ was able to fully restore

203 LacZ activities to that seen the parental $p f a A:: l a c Z$ strain relative to the vector only

204 control. Similarly, the ability to down regulate the operon in response to exogenous

205 Tween $80(18: 1)$ was restored in the complemented strain and not in the vector only

206 control (Figure 4B). Fatty acid analysis of the complemented strains indicated that

207 expression of $p f a F$ from this construct was able to fully restore EPA levels back to wild-

208 type levels compared to the vector only control (Table 3). Similarly, transcript 
209 abundances of $p f a A$ and $p f a D$ were restored to wild-type levels in the complemented

210 strain relative to vector only controls (data not shown).

211 Characterization of PfaF binding to pfa promoter

212 To verify that regulation by PfaF was direct, electrophoretic mobility shift assays

213 (EMSA) were conducted. PfaF was cloned onto pET28 with an N-terminal 6x-His-tag to

214 yield the construct pMA66 (Table 1). Using this construct, PfaF was expressed in E. coli

215 and purified to homogeneity by Ni-affinity chromatography (Supplementary Figure 1).

216 The DNA probe used in the EMSA was generated by PCR using a 6-carboxyfluorescein

217 (6-FAM) labeled primer set and was designed to cover 400 base pairs directly upstream

218 of the translational start of PfaA. As shown in Figure 5A, recombinant PfaF was able

219 bind to the $p f a A$ promoter probe in a concentration dependent manner forming a single

220 discrete complex. Inclusion of a non-FAM labeled competitor probe at approximately 80-

221 fold molar excess relative to the FAM labeled probe partially reversed the binding

222 indicating that the interaction between $\mathrm{PfaF}$ and the probe is specific (Figure 5B).

223 Further phylogenetic analyses of PfaF indicated that homologs exist in other

224 polyunsaturated fatty acid producing bacteria such as members of the genus Shewanella

225 and Colwellia, and that these homologs are distinct from other well known transcriptional

226 regulators associated with fatty acid biosynthesis (Supplemental Figure 2A). Further

227 investigation showed that that one such homolog from Shewanella amazonensis SB2B

228 (PBD: 3rh2) had an unpublished crystal structure (Supplemental Figure 2B). Analysis of

229 this crystal structure of the Shewanella PfaF homolog indicated that its C-terminal

230 domain contained a ligand pocket containing an unknown ligand resembling the

231 hydrocarbon "tail" of a fatty acid (Supplemental Figure 2C). Given the in vivo responses 
232 to the various fatty acid supplements observed, it was suspected that PfaF binding activity

233 could be mediated by oleoyl-CoA (18:1-CoA). Mobility shift assays indicated that the

234 addition of oleoyl-CoA abolished PfaF binding activity in a concentration dependent

235 manner (Figure 5C).

236 To localize the binding site for PfaF in the $p f a A$ promoter, non-radioactive DNase

237 I footprinting assays were conducted utilizing the 6-FAM labeled probe used in the

238 EMSA analysis. As shown in Figure 5D, inclusion of increasing amounts of purified PfaF

239 led to protection of a 22 base pair sequence (AGCACAACTGTGCATCGTCGAA)

240 corresponding to positions -182 to -204 of the promoter probe. The identified binding site

241 is positioned directly upstream of the previously identified -35 site within the $p f a A$

242 promoter (Figure 5E).

\section{Discussion}

245 In this work, the genetic regulation of the $p f a$ operon has been extensively

246 characterized utilizing a variety of genetic techniques. While the omega-3

247 polyunsaturated fatty acid products $(13,14)$, biosynthetic mechanism $(15,18,31)$, and

248 phylogenetic distribution $(16,32)$ of the bacterial pfa operon has been extensively

249 studied, there has been little work done describing how the operon is regulated and what

250 gene(s) might be involved. The findings described in this report represent the first

251 systematic investigation into the genetic regulation of the $p f a$ operon. The finding that

252 neither high hydrostatic pressure nor low temperature affected the activity of the

$253 p f a A:: l a c Z Y$ reporter is consistent with previously reported results in Photobacterium

254 profundum SS9 (25) and validated our reporter gene fusion approach. As noted 
255 previously in P. profundum SS9 (25), we confirmed the lack of correlation between the

256 expression level of the $p f a$ operon and the proportion of EPA found in the membrane

257 phospholipids under cold temperature and high pressure culture conditions. The reasons

258 for this phenomenon are unclear but they suggest that other factors in the biosynthesis

259 and membrane incorporation of EPA are involved in the increased abundance of EPA at

260 high pressure and/or cold temperature.

261 The finding that the $p f a$ operon was down regulated in response to exogenous

262 fatty acids in a FadR/FabR independent manner indicated that another transcription factor

263 was responsible for regulating this response to fatty acid supplementation. Screening of a

264 transposon library in the $p f a A:: l a c Z Y$ reporter strain identified a novel regulator, $p f a F$,

265 whose gene product acts as a positive regulator of the $p f a$ operon. Reintroduction of a

266 null mutation in $p f a F$ in SS9R yielded a mutant that had a specific several fold decrease

267 in EPA composition with relatively minor changes in the abundances of the other fatty

268 acids in the membrane. Successful complementation in trans confirmed the role of $p f a F$

269 in positive regulation of the $p f a$ operon. Based on amino acid sequence, $\mathrm{PfaF}$ is a member

270 of the TetR transcriptional regulator family, of which several members have been

271 characterized to be involved with regulation of fatty acid biosynthesis and/or degradation

272 in other bacteria (33).

273 Mobility shift and DNase I footprinting analyses verified that PfaF is capable of

274 binding to the $p f a A$ promoter and localized its binding site within the $p f a A$ promoter. The

275 binding site of $\mathrm{PfaF}$ and its position relative to the transcriptional start of $p f a A$ is typical

276 of other positive transcriptional regulators (34) and further supports the role of PfaF as

277 the transcriptional activator of the $p f a$ operon. The in vivo data indicated that PfaF 
278 regulates the $p f a$ operon in response to exogenous fatty acid supplementation, with the

279 most robust response mediated by a long-chain monounsaturated fatty acid (18:1). As

280 expected, addition of oleoyl-CoA disrupted PfaF binding activity in a concentration

281 dependent manner. Regulation of the operon in both SS9R and $p f a A:: l a c Z Y$ strains by

282 PfaF also indicates that the final EPA omega-3 fatty acid product of the Pfa synthase is

283 most likely not involved with mediating this regulatory activity. While the

284 physiologically relevant ligand(s) of PfaF has not been determined, the data presented in

285 this work strongly suggests that it is a fatty acid or a derivative thereof, e.g. acyl-CoA or

286 acyl-ACP.

287 Based on these results, a proposed model of regulation is shown in Figure 6. In

288 the absence of fatty acids, PfaF binds to its cognate sequence within the $p f a A$ promoter

289 region and acts as a positive regulator. In the presence of exogenous fatty acids, which

290 are presumably converted into acyl-CoA, PfaF binding to an acyl-CoA leads to its

291 dissociation from the promoter leading to a lack of transcriptional activation of the $p f a$

292 operon. Given its role in producing fatty acids for phospholipid biosynthesis (14) and its

293 utilization of the same precursor metabolites as the Type II fatty acid synthase (15), it is

294 not surprising that the Pfa synthase is controlled in a nearly identical fashion, albeit with

295 its own cognate regulatory protein.

296 Interestingly, genes regulated in a similar fashion, typically by FadR, have been

297 shown to be involved in processes related to virulence amongst various members of the

298 Vibrionaceae (35-38). Furthermore, many of these virulence genes are regulated in

299 response to exogenous fatty acids (39), similar to the results reported here for the $p f a$

300 operon. Whether the production of EPA or other PUFA is part of a virulence, symbiosis, 
301 or colonization response is unclear, although it is interesting to note that many PUFA-

302 producing bacteria have been isolated from the guts, glands, or other tissues of marine

303 animals such as fish and various invertebrates $(40,41)$.

304 At this time it is unclear as to how the various Tween derivatives and their fatty

305 acid components are utilized by P. profundum SS9. Various members of the

306 Vibrionaceae genus have been shown to have outer membrane associated lipase enzymes

307 capable of hydrolyzing esterified fatty acids for incorporation into phospholipids or usage

308 as a carbon source via $\beta$-oxidation $(42,43)$. In particular one such enzyme designated

309 VolA, has been well-characterized as being involved with utilization of exogenous

310 esterified fatty acids such as lysophosphotidylcholine (42). Preliminary searches of the $P$.

311 profundum SS9 genome located a homolog (locus tag: PBPRA2574) of volA in an operon

312 with a FadL homolog, identical to the genomic context of volA in Vibrio cholerae. This

313 lipase or other similar lipases may be involved with the utilization of Tween compounds

314 as a lipid source or as a carbon source.

315 Homology searches of available genomes indicated that all PUFA-producing

316 marine Gammaproteobacteria contain a $p f a F$ homolog many of which residing in similar

317 genomic contexts. Whether $p f a F$ is involved with regulating the $p f a$ operon in these

318 strains has yet to be determined. In many instances, such as in strains of Shewanella and

319 Colwellia, the $p f a$ operon also contains an annotated regulator typically designated as

$320 p f a R$, immediately upstream of $p f a A$. Interestingly, the protein sequence of this regulator

321 does not match to any class of bacterial transcriptional regulators and only contains an

322 identifiable N-terminal helix-turn-helix domain, which is most likely involved in DNA-

323 binding activity. Preliminary results using S. piezotolerans WP3, a genetically tractable 
324 EPA producer, indicated no differences in EPA composition between wild-type and

$325 \Delta p f a R$ mutants under a variety of temperatures (data not shown). A previous study (44)

326 demonstrated that replacing $p f a R$ with an inducible promoter could lead to dramatic

327 increases in EPA production in a heterologous host strain of E.coli. Unfortunately, that

328 study lacked adequate data that could be used to ascertain the role of $p f a R$ directly.

329 Regulation of the $p f a$ operon in strains with $p f a R$ may indeed be more complex or

330 otherwise different than in the case of P. profundum SS9.

331 The results presented here describe the identification of a novel transcriptional

332 regulator in the model marine bacterium P. profundum SS9 that specifically modulates

333 expression of the $p f a$ operon in response to exogenous fatty acids and controls the amount

334 of polyunsaturated fatty acid incorporated into membrane phospholipids. This study adds

335 new insight into the unique lipid physiology of widespread marine bacteria and offers

336 new opportunities for the genetic optimization of microbial omega-3 polyunsaturated

337 fatty acid synthesis.

339 Materials and Methods

\section{Bacterial strains and growth conditions}

341 Escherichia coli strains were routinely grown at $37^{\circ} \mathrm{C}$ in Luria Bertani (LB)

342 media unless stated otherwise. Photobacterium profundum SS9 strains were grown at

$34315^{\circ} \mathrm{C}$ in 2216 marine broth (Difco) at $75 \%$ strength $(28 \mathrm{~g} / \mathrm{L})$ unless noted otherwise. For

344 solid medias, agar was included at $15 \mathrm{~g} / \mathrm{L}$. The antibiotics kanamycin $(50 \mu \mathrm{g} / \mathrm{ml}$ for E. coli

345 and $200 \mu \mathrm{g} / \mathrm{ml}$ for $P$. profundum $)$, chloramphenicol $(15 \mu \mathrm{g} / \mathrm{ml})$, ampicillin $(100 \mu \mathrm{g} / \mathrm{ml})$,

346 and rifampicin $(100 \mu \mathrm{g} / \mathrm{ml})$ were used as required. For high-pressure growth experiments, 
347 P. profundum SS9 strains were grown in heat-sealed bulbs and incubated in stainless steel

348 pressure vessels as described previously (45).

\section{Targeted Mutagenesis}

$351 \quad$ Vectors for introducing mutations into $P$. profundum were introduced by

352 conjugation using previously described methods with minor alterations $(14,45)$. In-frame

353 deletions were generated by allelic exchange using the suicide vector pRE118 (46).

354 Insertional inactivation of target genes was accomplished by introduction of the suicide

355 plasmid pMUT100 as described previously $(23,47)$.

\section{Transposon mutagenesis and screening}

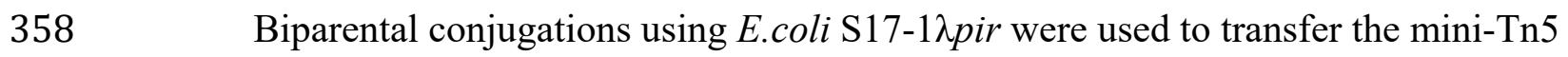

359 delivery plasmid pRL27 into the $p f a A:: l a c Z Y$ reporter strain (Table 1) $(29,48)$. Both

360 recipient and donor strains were grown to stationary phase and conjugations were

361 performed as described above. After $\sim 24 \mathrm{hr}$ at ambient temperature $\left(\sim 22^{\circ} \mathrm{C}\right)$ cells on filter

362 membranes were resuspended in 2216 broth and plated onto selection media (2216 agar

363 containing $200 \mu \mathrm{g} / \mathrm{ml}$ kanamycin and $100 \mu \mathrm{g} / \mathrm{ml}$ rifampin) and incubated at $15^{\circ} \mathrm{C}$ for 5

364 days. Resulting ex-conjugants were patched to fresh selection plates in grid format. After

365 two days growth at $15^{\circ} \mathrm{C}$ the arrayed mutants were replica plated to 2216 agar with X-gal

$366(80 \mu \mathrm{g} / \mathrm{ml})$. After two days of growth on indicator media, mutants were screened by eye

367 for differences in blue colony formation. Mutants with differential lacZ activity were

368 clonally isolated and further screened by $\beta$-galactosidase assays in liquid cultures as

369 described below. 


\section{Identification of transposon insertion sites}

372 To identify transposon insertion sites of interest an arbitrary PCR method was

373 utilized similar to the method described previously (30). Primer sequences are given in

374 Supplementary Table 1. In the first round of PCR, a primer specific to one end of the

375 mini Tn5 element (Tn5 ext) in combination with one of three degenerate primers (arb1, 2,

376 or 3 ) is used with purified genomic DNA as a template. The conditions used for the first

377 PCR were; $95^{\circ} \mathrm{C} 5 \mathrm{~min}, 6$ cycles of $95^{\circ} \mathrm{C}$ for $30 \mathrm{sec}, 30^{\circ} \mathrm{C}$ for $30 \mathrm{sec}, 68^{\circ} \mathrm{C}$ for $2 \mathrm{~min}$,

378 followed by 30 cycles of $95^{\circ} \mathrm{C}$ for $30 \mathrm{sec}, 45^{\circ} \mathrm{C}$ for $30 \mathrm{sec}, 68^{\circ} \mathrm{C} 2 \mathrm{~min}$, and $68^{\circ} \mathrm{C} 5 \mathrm{~min}$.

379 Two microliters of the first reaction was used as a template for a nested PCR with "Arb

380 clamp" and "Tn5 int" primers. The conditions for the second PCR were $95^{\circ} \mathrm{C}$ for $5 \mathrm{~min}$,

381 followed by 30 cycles of $95^{\circ} \mathrm{C}$ for $30 \mathrm{sec}, 55^{\circ} \mathrm{C}$ for $30 \mathrm{sec}, 68^{\circ} \mathrm{C}$ for $2 \mathrm{~min}$, followed by

$38268^{\circ} \mathrm{C}$ for $10 \mathrm{~min}$. PCR reactions yielding single amplicons as judged by agarose gel

383 electrophoresis were purified using a PCR clean up kit (Zymo Research) and sent for

384 DNA sequencing. Sequences were compared to the genome of P. profundum SS9 to

385 determine the insertion site of the mini-Tn5 element.

$\beta$-galactosidase assays

388 Cultures of indicated strains were grown at $15^{\circ} \mathrm{C}$ in aerobic tubes, unless noted 389 otherwise. Mid-log phase cultures $\left(\mathrm{OD}_{600}=0.2-0.6\right)$ were assayed for changes in LacZ

390 activity from whole cell extracts using the SDS and chloroform lysis modification

391 described previously (49). Activities reported are in Miller units and represent the mean 392 of at least five independent experiments. 


\section{RNA isolation and quantitative reverse transcriptase PCR (qRT-PCR)}

395 Total RNA was isolated from mid-log phase cells grown under the indicated 396 conditions using Trizol (Invitrogen) following manufacturer guidelines. Crude RNA 397 extracts were further purified and treated with DNase I (Zymo Research) using the RNA

398 Clean and Concentrator kit (Zymo Research). For cDNA synthesis, the Superscript III

399 First Strand synthesis kit (Invitrogen) was used following manufacturer's recommended

400 protocols. Quantitative PCR's were performed using the Maxima Sybr Green Master Mix

401 (Thermo Scientific) and run on a Stratagene MX3000P qPCR system. For quantification

402 of target transcripts, the gyrB gene (PBPRA0011) was used as an internal reference and

403 differences in expression were calculated using the $\Delta \Delta \mathrm{CT}$ method. Primers for qPCR

404 experiments are listed in Supplementary Table 1.

405

\section{Expression and Purification of PfaF}

407 PfaF (locus tag: PBPRA0221) was cloned into pET28 as an NheI-XhoI fragment

408 (Novagen) as to generate $\mathrm{N}$-terminal 6xHis tagged protein. After sequence verification,

409 this construct was transformed into BL21 DE3 Tuner pLysS (Novagen) cells following

410 standard procedures (49). For protein expression, overnight cultures were diluted 1/100

411 into LB supplemented with chloramphenicol $(30 \mu \mathrm{g} / \mathrm{ml})$ and kanamycin $(50 \mu \mathrm{g} / \mathrm{ml})$ and

412 grown at $30^{\circ} \mathrm{C}$ until $\mathrm{OD}_{600}$ of $\sim 0.5$ at which point IPTG was added to final concentration

413 of $0.5 \mathrm{mM}$ and grown for an additional $4 \mathrm{hrs}$ at $30^{\circ} \mathrm{C}$. Cells were harvested by

414 centrifugation and cell pellets were processed or stored at $-80^{\circ} \mathrm{C}$. Frozen cell pellets were

415 thawed on ice with the addition of buffer A (50mM Tris-Cl pH 7.5, 200mM NaCl, 10\% 
416 glycerol). Lysozyme was added and cells incubated on ice for 30 minutes and sonicated

417 on ice to complete the lysis procedure. The lysate was centrifuged at $10,000 \mathrm{rpm}, 4^{\circ} \mathrm{C}$, for

41830 minutes to separate insoluble and soluble fractions. The clarified supernatant was

419 applied to a Ni-NTA column equilibrated with buffer $\mathrm{A}$ and mixed gently at $4^{\circ} \mathrm{C}$ for one

420 hour. The resin was washed with several column volumes of buffer B (buffer A $+30 \mathrm{mM}$

421 imidazole) and proteins were eluted with buffer $\mathrm{C}$ (buffer $\mathrm{A}+300 \mathrm{mM}$ imidazole). Eluted

422 fractions were checked by SDS-PAGE for purity, and appropriate fractions were pooled

423 and desalted using PD-10 columns (GE Healthcare) and exchanged into Buffer D (20mM

424 Tris $\mathrm{Cl} \mathrm{pH} 7.5,50 \mathrm{mM} \mathrm{NaCl}, 10 \%$ Glycerol). Protein samples were pooled and

425 subsequently concentrated by 10,000 kDa centrifugal filter units (Amicon).

426

427 Electrophoretic mobility shift assays and DNase I footprinting

428 A 400bp DNA fragment, which included the previously mapped promoter of

$429 p f a A$, was generated by PCR using a 6-carboxyfluorescein (FAM) labeled primer listed in

430 Supplemental Table 1 and purified P. profundum SS9 genomic DNA as a template. For

431 mobility shift experiments, binding reactions contained; binding buffer (20mM Tris-Cl

$432 \mathrm{pH} 7.5,0.2 \mathrm{mg} / \mathrm{ml} \mathrm{BSA}, 0.5 \mathrm{mM} \mathrm{CaCl}_{2}, 2.5 \mathrm{mM} \mathrm{MgCl}_{2}, 10 \%$ glycerol), $2 \mu \mathrm{g}$ poly dI-dC

433 (Thermo Scientific), 25nM promoter probe, and the indicated amount of purified PfaF.

434 Binding reactions were incubated at $22^{\circ} \mathrm{C}$ for $60 \mathrm{~min}$ and analyzed by electrophoresis

435 using pre-run $6 \% 0.5 \mathrm{X}$ TBE, $1 \%$ glycerol, native polyacrylamide gels (50). Gels were

436 visualized and photographed using a GelDoc system (Bio-Rad).

437 Non-radioactive DNase I footprinting (51) was performed using the same binding

438 buffer and reaction conditions used in the EMSA experiments described above. Digests 
439 were initiated by the addition of $0.03 \mathrm{U}$ of DNase I (NEB Biolabs), and incubated at $22^{\circ} \mathrm{C}$

440 for 2 minutes. Digestion reactions were stopped by the addition of DNase I stop buffer

441 (NEB) and were extracted with phenol:chloroform:isoamyl alcohol (25:24:1, Thermo

442 Scientific). DNA fragments were further purified by use of a PCR purification kit

443 (Zymo). Eluted DNA fragments were subjected to fragment analysis by capillary

444 electrophoresis (Eton BioScience). Chromatograms were examined using the

445 microsatellite plugin within Geneious Prime 2019.2.3 (https://www.geneious.com).

446 Protected regions were identified and compared to the included LIZ-500 size standards to

447 identify the binding site coordinates and relevant protected bases.

\section{Fatty acid extraction and GC-MS analysis}

450 Late log phase cultures were harvested by centrifugation and cell pellets rinsed

451 once with $50 \%$ Sigma Sea Salts solution $(16 \mathrm{~g} / \mathrm{L})$ and stored at $-80^{\circ} \mathrm{C}$. Cell pellets were

452 lyophilized and fatty acids were converted to fatty acid methyl esters and analyzed by gas

453 chromatography mass spectrometry using previously described protocols and methods

454 (14).

\section{Acknowledgements}

457 We would like to thank Dr. Doug Bartlett for insightful discussions regarding

458 genetic manipulations in P. profundum SS9 and Dr. Bianca Brahamsha for the generous

459 gift of the pRL27 mini-Tn5 transposon delivery vector. This work was supported by

460 National Science Foundation Division of Molecular and Cellular Biosciences grant

461 MCB-1149552 to E.E.A. 


\section{References}

464 1. Parsons JB, Rock CO. 2013. Bacterial lipids: Metabolism and membrane 465 homeostasis. Prog Lipid Res 52:249-276.

466 2. Zhu K, Zhang Y-M, Rock CO. 2009. Transcriptional Regulation of Membrane

467 Lipid Homeostasis in Escherichia coli. J Biol Chem 284:34880-34888.

468 3. Feng Y, Cronan JE. 2011. Complex binding of the FabR repressor of bacterial

469 unsaturated fatty acid biosynthesis to its cognate promoters. Mol Microbiol

$470 \quad \mathbf{8 0}: 195-218$.

471 4. Cronan JE, Subrahmanyam S. 1998. FadR, transcriptional co-ordination of 472 metabolic expediency. Mol Microbiol 29:937-43.

473 5. Magnuson K, Jackowski S, Rock CO, Cronan JE. 1993. Regulation of fatty 474 acid biosynthesis in Escherichia coli. Microbiol Rev 57:522-42.

475 6. Henry MF, Cronan JE. 1992. A new mechanism of transcriptional regulation:

476 release of an activator triggered by small molecule binding. Cell 70:671-679.

477 7. Campbell J, Cronan JE. 2001. Escherichia coli FadR positively regulates

478 transcription of the fabB fatty acid biosynthetic gene. J Bacteriol 183:5982-5990.

479 8. Zhang Y-M, Marrakchi H, Rock CO. 2002. The FabR (YijC) transcription 480 factor regulates unsaturated fatty acid biosynthesis in Escherichia coli. J Biol $481 \quad$ Chem 277:15558-65.

482 9. Luo Q, Shi M, Ren Y, Gao H. 2014. Transcription factors FabR and FadR

483 regulate both aerobic and anaerobic pathways for unsaturated fatty acid

484 biosynthesis in Shewanella oneidensis. Front Microbiol 5:1-10. 
485 10. Zhu K, Choi K-H, Schweizer HP, Rock CO, Zhang Y-M. 2006. Two aerobic

486 pathways for the formation of unsaturated fatty acids in Pseudomonas aeruginosa.

$487 \quad$ Mol Microbiol 60:260-73.

488 11. Zhang Y-M, Zhu K, Frank MW, Rock CO. 2007. A Pseudomonas aeruginosa

489 transcription factor that senses fatty acid structure. Mol Microbiol 66:622-32.

490 12. Subramanian C, Rock CO, Zhang YM. 2010. DesT coordinates the expression

491 of anaerobic and aerobic pathways for unsaturated fatty acid biosynthesis in

492 Pseudomonas aeruginosa. J Bacteriol 192:280-285.

493 13. Yoshida K, Hashimoto M, Hori R, Adachi T, Okuyama H, Orikasa Y,

494 Nagamine T, Shimizu S, Ueno A, Morita N. 2016. Bacterial long-chain

495 polyunsaturated fatty acids: Their biosynthetic genes, functions, and practical use.

$496 \quad$ Mar Drugs 14.

497 14. Allemann MN, Allen EE. 2018. Characterization and Application of Marine

498 Microbial Omega-3 Polyunsaturated Fatty Acid Synthesis. Methods Enzymol, 1st

499 ed. 605:3-32.

500 15. Metz JG, Roessler P, Facciotti D, Levering C, Dittrich F, Lassner M,

501 Valentine RC, Lardizabal K, Domergue F, Yamada A, Yazawa K, Knauf V,

502 Browse J. 2001. Production of polyunsaturated fatty acids by polyketide synthases

503 in both prokaryotes and eukaryotes. Science 293:290-3.

504 16. Shulse CN, Allen EE. 2011. Widespread occurrence of secondary lipid

505 biosynthesis potential in microbial lineages. PLoS One 6:e20146.

506 17. Cronan JE, Rock CO. 2008. Biosynthesis of Membrane Lipids. EcoSal Plus 3.

507 18. Santín O, Moncalián G. 2018. Loading of malonyl-CoA onto tandem acyl carrier 
$509 \quad 12501$.

510 19. Cho H-N, Kasai W, Kawamoto J, Esaki N, Kurihara T. 2012. Characterization

511 of 1-acyl-sn-glycerol-3-phosphate acyltransferase from a polyunsaturated fatty

512 acid-producing bacterium, Shewanella livingstonensis Ac10. Trace Nutr Res

513 29:92-99.

514 20. Yao J, Rock CO. 2012. Phosphatidic acid synthesis in bacteria. Biochim Biophys

$515 \quad$ Acta - Mol Cell Biol Lipids 1831:495-502.

516 21. Toyotake Y, Cho HN, Kawamoto J, Kurihara T. 2018. A novel 1-acyl-sn-

517 glycerol-3-phosphate O-acyltransferase homolog for the synthesis of membrane

518 phospholipids with a branched-chain fatty acyl group in Shewanella

519 livingstonensis Ac10. Biochem Biophys Res Commun 500:704-709.

520 22. Ogawa T, Tanaka A, Kawamoto J, Kurihara T. 2018. Purification and

521 characterization of 1-acyl-sn-glycerol-3-phosphate acyltransferase with a substrate

522 preference for polyunsaturated fatty acyl donors from the eicosapentaenoic acid-

523 producing bacterium Shewanella livingstonensis Ac10. J Biochem 164:33-39.

524 23. Allen EE, Facciotti D, Bartlett DH. 1999. Monounsaturated but not

525 polyunsaturated fatty acids are required for growth of the deep-sea bacterium

526 Photobacterium profundum SS9 at high pressure and low temperature. Appl

$527 \quad$ Environ Microbiol 65:1710-20.

528 24. Allen EE, Bartlett DH. 2000. FabF Is Required for Piezoregulation of cis-

529 Vaccenic Acid Levels and Piezophilic Growth of the Deep-Sea Bacterium

530 Photobacterium profundum Strain SS9. J Bacteriol 182:1264-1271. 
531 25. Allen EE, Bartlett DH. 2002. Structure and regulation of the omega-3

532 polyunsaturated fatty acid synthase genes from the deep-sea bacterium

533 Photobacterium profundum strain SS9. Microbiology 148:1903-1913.

534 26. Wang F, Xiao X, Ou H-Y, Gai Y, Wang F. 2009. Role and regulation of fatty

535 acid biosynthesis in the response of Shewanella piezotolerans WP3 to different

536 temperatures and pressures. J Bacteriol 191:2574-84.

537 27. Kawamoto J, Kurihara T, Yamamoto K, Nagayasu M, Tani Y, Mihara H,

538 Hosokawa M, Baba T, Sato SB, Esaki N. 2009. Eicosapentaenoic Acid Plays a

539 Beneficial Role in Membrane Organization and Cell Division of a Cold-Adapted

$540 \quad$ Bacterium, Shewanella livingstonensis Ac10. J Bacteriol 191:632-640.

541 28. Valentine RC, Valentine DL. 2004. Omega-3 fatty acids in cellular membranes:

542 A unified concept. Prog Lipid Res 43:383-402.

543 29. Larsen RA, Wilson MM, Guss AM, Metcalf WW. 2002. Genetic analysis of

544 pigment biosynthesis in Xanthobacter autotrophicus Py2 using a new, highly

545 efficient transposon mutagenesis system that is functional in a wide variety of

546 bacteria. Arch Microbiol 178:193-201.

547 30. Lauro FM, Tran K, Vezzi A, Vitulo N, Valle G, Bartlett DH. 2008. Large-scale

548 transposon mutagenesis of Photobacterium profundum SS9 reveals new genetic

549 loci important for growth at low temperature and high pressure. J Bacteriol

550 190:1699-709.

551 31. Jiang H, Zirkle R, Metz JG, Braun L, Richter L, Van Lanen SG, Shen B.

552 2008. The role of tandem acyl carrier protein domains in polyunsaturated fatty acid

553 biosynthesis. J Am Chem Soc 130:6336-7. 
554 32. Shulse CN, Allen EE. 2011. Diversity and distribution of microbial long-chain

555 fatty acid biosynthetic genes in the marine environment. Environ Microbiol

$556 \quad 13: 684-95$.

557 33. Cuthbertson L, Nodwell JR. 2013. The TetR Family of Regulators. Microbiol

$558 \quad$ Mol Biol Rev 77:440-475.

559 34. Browning DF, Busby SJW. 2004. The regulation of bacterial transcription

560 initiation. Nat Rev Microbiol 2:57-65.

561 35. Brown RN, Gulig PA. 2008. Regulation of fatty acid metabolism by FadR is

562 essential for Vibrio vulnificus to cause infection of mice. J Bacteriol 190:7633-

5637644.

564 36. Kovacikova G, Lin W, Taylor RK, Skorupski K. 2017. The fatty acid regulator

$565 \quad$ FadR influences the expression of the virulence cascade in the El Tor biotype of

$566 \quad$ Vibrio cholerae by modulating the levels of ToxT via two different mechanisms. J

$567 \quad$ Bacteriol 199:1-16.

568 37. Feng Y, Cronan JE. 2011. The Vibrio cholerae fatty acid regulatory protein,

569 FadR, represses transcription of plsB, the gene encoding the first enzyme of

570 membrane phospholipid biosynthesis. Mol Microbiol 81:1020-33.

571 38. Gao R, Li D, Lin Y, Lin J, Xia X, Wang H, Bi L, Zhu J, Hassan B, Wang S,

572 Feng Y. 2017. Structural and Functional Characterization of the FadR Regulatory

573 Protein from Vibrio alginolyticus. Front Cell Infect Microbiol 7:1-16.

574 39. Hung DT, Mekalanos JJ. 2005. Bile acids induce cholera toxin expression in $575 \quad$ Vibrio cholerae in a ToxT-independent manner. Proc Natl Acad Sci 102:3028$576 \quad 3033$. 
577 40. Okuyama H, Orikasa Y, Nishida T, Watanabe K, Morita N. 2007. Bacterial

578 genes responsible for the biosynthesis of eicosapentaenoic and docosahexaenoic

579 acids and their heterologous expression. Appl Environ Microbiol 73:665-670.

580 41. Yano Y, Nakayama A, Yoshida K. 1997. Distribution of polyunsaturated fatty

$581 \quad$ acids in bacteria present in intestines of deep-sea fish and shallow-sea

582 poikilothermic animals. Appl Environ Microbiol 63:2572-2577.

583 42. Pride AC, Herrera CM, Guan Z, Giles DK, Trent MS. 2013. The outer surface

584 lipoprotein VolA mediates utilization of exogenous lipids by Vibrio cholerae.

$585 \quad$ MBio 4:1-9.

586 43. Giles DK, Hankins J V., Guan Z, Trent MS. 2011. Remodelling of the Vibrio

587 cholerae membrane by incorporation of exogenous fatty acids from host and

$588 \quad$ aquatic environments. Mol Microbiol 79:716-728.

589 44. Lee S-J, Kim CH, Seo P-S, Kwon O, Hur B-K, Seo J-W. 2008. Enhancement of

590 heterologous production of eicosapentaenoic acid in Escherichia coli by

591 substitution of promoter sequences within the biosynthesis gene cluster.

$592 \quad$ Biotechnol Lett 30:2139-2142.

593 45. Chi E, Bartlett DH. 1993. Use of a reporter gene to follow high pressure signal

594 transduction in the deep-sea bacterium Photobacterium sp.strain SS9. J Bacteriol

$595 \quad \mathbf{1 7 5 : 7 5 3 3 - 7 5 4 0 .}$

596 46. Edwards RA, Keller LH, Schifferli DM. 1998. Improved allelic exchange

597 vectors and their use to analyze 987P fimbria gene expression. Gene 207:149-57.

598 47. Brahamsha B. 1996. A genetic manipulation system for oceanic cyanobacteria of 599 the genus Synechococcus. Appl Environ Microbiol 62:1747-51. 
600 48. Simon R, Priefer U, Pühler A. 1983. A Broad Host Range Mobilization System

601 for In Vivo Genetic Engineering: Transposon Mutagenesis in Gram Negative

$602 \quad$ Bacteria. Bio/Technology 1:784-791.

603 49. Miller JH. 1992. A short course in bacterial genetics: a laboratory manual and

604 handbook for Escherichia coli and related bacteria. Cold Spring Harbor Laboratory

605 Press.

606 50. Carey MF, Peterson CL, Smale ST. 2013. Electrophoretic Mobility-Shift

607 Assays. Cold Spring Harb Protoc 8:636-639.

608 51. Zianni M, Tessanne K, Merighi M, Laguna R, Tabita FR. 2006. Identification

609 of the DNA bases of a DNase I footprint by the use of dye primer sequencing on

610 an automated capillary DNA analysis instrument. J Biomol Tech 17:103-13.

611 52. Saltikov CW, Newman DK. 2003. Genetic identification of a respiratory arsenate 612 reductase. Proc Natl Acad Sci 100:10983-10988.

613 53. Lauro FM, Eloe EA, Liverani N, Bertoloni G, Bartlett DH. 2005. Conjugal

614 vectors for cloning, expression, and insertional mutagenesis in gram-negative

615 bacteria. Biotechniques 38:708-712.

616 54. Better M, Helinski DR. 1983. Isolation and characterization of the recA gene of 617 Rhizobium meliloti. J Bacteriol 155:311-6.

\section{$619 \quad$ Figure legends}

620

621 Figure 1. Genetic regulation of monounsaturated fatty acid biosynthesis genes fabA and $622 f a b B$ in E. coli. In the absence of exogenous fatty acids FadR binds to a site upstream of 
623 the $f a b A$ and $f a b B$ promoters and acts as an activator of transcription. When present,

624 exogenous fatty acids are transported across the outer membrane by FadL and converted

625 into acyl-CoA by FadD. Acyl-CoA binding to FadR causes a conformational shift that

626 abolishes the DNA binding capabilities of FadR. In both scenarios FabR binds to a site

627 downstream of FadR and has been shown to bind in the presence/absence of acyl-CoA

628 and/or Acyl-ACP. Loss of FadR activation of transcription presumably allows FabR to

629 act as a better repressor of $f a b A / B$ expression.

630

631 Figure 2. Reporter gene expression in the $p f a A:: l a c Z Y$ strain in response to a variety of

632 culture parameters known to modulate EPA composition. (A) Various temperature and

633 hydrostatic pressure conditions. (B) Cells cultured at $15^{\circ} \mathrm{C}$ with $0.05 \%$ Tween compound

634 supplement indicated. For (A) and (B) results of at least six independent experiments

635 shown as means with error bars representing standard deviation (ns, $P>0.05 ; * * *, P$

$636<0.005$; ****, $P<0.0001$ ). (C) Effect of $0.05 \%$ Tween 80 supplementation on various

637 fatty acid biosynthetic gene transcript abundances in SS9R as determined by qRT-PCR.

638 Cells grown without supplementation represent the calibrator condition. Error bars

639 represent the standard deviations based on at least three independent biological replicates

640 with duplicate qPCR reactions $(*, P<0.05 ; * *, P<0.005)$.

641

642 Figure 3. Influence of FadR and FabR on expression of the $p f a$ operon. (A) LacZ

643 activities of strains carrying $\Delta f a b R$ and/or $\Delta f a d R$ mutations in the presence or absence of

$6440.05 \%$ Tween $80(18: 1)$. Results of at least six independent experiments shown as means

645 with error bars representing standard deviation. (**, $P<0.05 ; * * *, P<0.005 ; * * * *, P$ 
$646<0.0001$ ) (B) Relative transcript abundances of $p f a A$, $p f a D, f a b A$, and $f a b B$ grown in the

647 presence or absence of Tween $80(18: 1)$ in (SS9R $\Delta f a b R \Delta f a d R)$. Cells grown without

648 supplement represent the calibrator condition. Error bars represent the standard

649 deviations based on at least three independent biological replicates with duplicate qPCR

650 reactions $(\mathrm{NS}, P>0.05 ; * * *, P<0.005)$.

651

652 Figure 4. The $p f a F$ genetic locus and regulatory phenotypes associated with its

653 disruption. (A) Genetic organization of PfaF locus, arrow indicates relative position of

654 mini-Tn5 insertion site in the $p f a A:: l a c Z Y$ regulatory mutant. (B) LacZ activity of

$655 p f a F::$ Tn5 mutant and the complemented strains carrying pMA62 (pFL122 pfaF) or

656 vector (pFL122) only $\pm 0.05 \%$ Tween 80 (18:1) supplementation (ns, $P>0.05 ; * * * *, P$

$657<0.0001$ ). (C) qRT-PCR analysis of $p f a A$, $p f a D$, fabA, and $f a b B$ transcript abundances in

658 SS9R $p f a F$ mutant relative to SS9R. Error bars represent the standard deviations based on

659 at least three independent biological replicates with duplicate qPCR reactions (ns, $P$

$660>0.05 ; * *, P<0.005 ; * * *, P<0.001)$.

661

662 Figure 5. Characterization of PfaF binding to the $p f a A$ promoter. (A) Electrophoretic

663 mobility shift assay demonstrating PfaF binding to the $p f a A$ promoter in a concentration

664 dependent manner. (B) Binding of FAM-labeled probe (+FAM) is partially inhibited by

665 the inclusion of molar excess of unlabeled probe (-FAM) indicating that PfaF binding is

666 specific. (C) Addition of oleoyl-CoA at the indicated concentrations reverses the binding

667 of PfaF to the probe in a concentration dependent manner. (D) DNase I footprinting

668 analysis of PfaF binding to $p f a A$ promoter. Purified PfaF was added at the indicated 
669 concentrations and subjected to DNase I digestion as described in Materials and Methods.

670 Chromatograms and sequencing traces shown correspond to the coding strand and the

671 box indicates the region protected from digestion by PfaF. (E) DNA sequence of $p f a A$

672 probe used in mobility shift and footprinting assays. Putative promoter elements (-35 and

$673-10$ sites) and transcriptional start site (arrow) were previously determined (25). Region

674 protected by PfaF indicated in bold, green, underlined font.

675

676 Figure 6. Proposed model of regulation of the $p f a$ operon as mediated by PfaF. In the

677 absence of fatty acids PfaF binds to the $p f a A$ promoter and acts as a positive regulator. In

678 the presence of exogenous fatty acids PfaF binds to acyl-CoA and releases from the

679 promoter region leading to down regulation of the $p f a$ operon.

680

681 Table 1. Strains and Plasmids used in this study

\begin{tabular}{|c|c|c|}
\hline Strain/Plasmid & Relevant characteristics & Source \\
\hline \multicolumn{3}{|l|}{ E.coli strains } \\
\hline MG1655 & Wild type E.coli, source of lacZY operon & $\begin{array}{l}\text { Coli Genetic } \\
\text { Stock Center }\end{array}$ \\
\hline DH5apir & Cloning strain for pir vectors & $(52)$ \\
\hline S17-1גpir & Biparental mating strain & $(48)$ \\
\hline \multicolumn{3}{|c|}{ Photobacterium profundum strains } \\
\hline SS9R & Rifampin resistant derivative of $P$. profundum SS9 & $(45)$ \\
\hline EA2 & EPA overproducer, $p f a$ regulatory mutant & $(23)$ \\
\hline MAP7 & SS9R, $\Delta l a c Z$ & This study \\
\hline MAP12 & $\mathrm{SS} 9 \mathrm{R}, \Delta f a b R$ & This study \\
\hline MAP13 & $\mathrm{SS} 9 \mathrm{R}, \Delta \mathrm{fadR}$ & This study \\
\hline MAP15 & $\mathrm{SS} 9 \mathrm{R}, \Delta f a b R \Delta$ fadR & This study \\
\hline MAP16 & MAP7, $\triangle p f a A:: l a c Z Y$ & This study \\
\hline MAP17 & MAP $16, \Delta f a b R$ & This study \\
\hline MAP18 & MAP16, $\Delta$ fadR & This study \\
\hline MAP23 & MAP $16, \Delta f a d R \Delta f a b R$ & This study \\
\hline MAP26 & MAP16, pfaF::pMUT100 & This study \\
\hline MAP27 & SS9R, pfaF::pMUT100 & This study \\
\hline
\end{tabular}




\begin{tabular}{|c|c|c|}
\hline MAP1603 & MAP16, $p f a F:: \operatorname{Tn} 5$ & This study \\
\hline Plasmids & & \\
\hline pRE118 & Allelic exchange vector $\mathrm{Kan}^{\mathrm{R}}$ & $(46)$ \\
\hline pFL122 & Broad host range complementation vector & $(53)$ \\
\hline pRK2073 & Conjugation helper plasmid & $(54)$ \\
\hline pMUT100 & Suicide plasmid for insertional inactivation & $(47)$ \\
\hline pRL27 & Mini Tn5 transposon delivery vector & $(29)$ \\
\hline pET28 & T7 expression plasmid & Novagen \\
\hline pMA21 & pRE118 w/ $/ p f a A$ allele & This study \\
\hline pMA29 & pRE118 w/AlacZ allele & This study \\
\hline pMA38 & pRE118 w/ $f a b R$ allele & This study \\
\hline pMA39 & $\mathrm{pRE} 118 \mathrm{w} / \Delta$ fadR allele & This study \\
\hline pMA41 & pRE118 w/ $\triangle p f a A:: l a c Z Y$ allele & This study \\
\hline pMA61 & pMUT100 w/pfaF internal fragment & This study \\
\hline pMA62 & $\mathrm{pFL} 122 \mathrm{w} / p f a F$ & This study \\
\hline pMA66 & pET28 w/PfaF N-terminal $6 x$ His tag & This study \\
\hline
\end{tabular}

683 Table 2. Fatty acid compositions of SS9R and various $p f a F$ mutant strains at $15^{\circ} \mathrm{C}$

\begin{tabular}{|lcccc|}
\hline \multicolumn{5}{c|}{ Mean \% fatty acid by weight $^{\mathbf{a}}$} \\
Fatty acid & SS9R & pfaF & pfaF pFL122 & pfaF pMA62 \\
& $4.16 \pm 1.53$ & $2.84 \pm 0.33$ & $3.72 \pm 0.45$ & $3.73 \pm 0.10$ \\
$\mathbf{1 2 : 0}$ & $4.33 \pm 1.21$ & $5.25 \pm 0.94$ & $9.46 \pm 0.31$ & $8.03 \pm 0.22$ \\
$\mathbf{1 4 : 0}$ & $3.19 \pm 1.11$ & $3.90 \pm 0.73$ & $6.04 \pm 0.19$ & $4.90 \pm 0.16$ \\
$\mathbf{1 4 : 1}$ & $21.07 \pm 1.84$ & $19.03 \pm 0.67$ & $19.39 \pm 3.64$ & $19.50 \pm 2.82$ \\
$\mathbf{1 6 : 0}$ & $45.09 \pm 1.68$ & $50.95 \pm 0.53$ & $45.33 \pm 2.06$ & $39.59 \pm 1.58$ \\
$\mathbf{1 6 : 1}$ & $1.99 \pm 1.23$ & $2.22 \pm 0.28$ & $2.66 \pm 0.14$ & $2.96 \pm 0.27$ \\
$\mathbf{1 2 - O H}$ & $0.71 \pm 0.11$ & $0.94 \pm 0.08$ & $1.37 \pm 0.15$ & $1.74 \pm 0.09$ \\
$\mathbf{1 8 : 0}$ & $12.26 \pm 2.26$ & $12.92 \pm 0.42$ & $9.38 \pm 0.97$ & $12.02 \pm 2.82$ \\
$\mathbf{1 8 : 1}$ & $4.52 \pm 1.55$ & $0.82 \pm 0.23$ & $1.14 \pm 0.17$ & $5.14 \pm 0.30$ \\
$\mathbf{2 0 : 5}$ & & &
\end{tabular}

$684{ }^{\mathrm{a}}$ Data represents the average of at least three independent cultures \pm standard deviation

685

686

687 
bioRxiv preprint doi: https://doi.org/10.1101/2020.01.28.924217; this version posted January 29, 2020. The copyright holder for this preprint (which was not certified by peer review) is the author/funder. All rights reserved. No reuse allowed without permission.

\section{Figure 1}

\section{Absence of exogenous fatty acids}

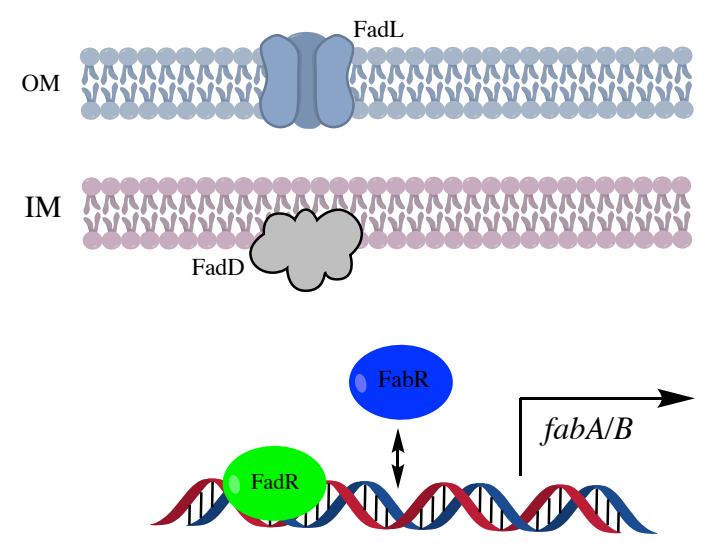

\section{Presence of exogenous fatty acids}

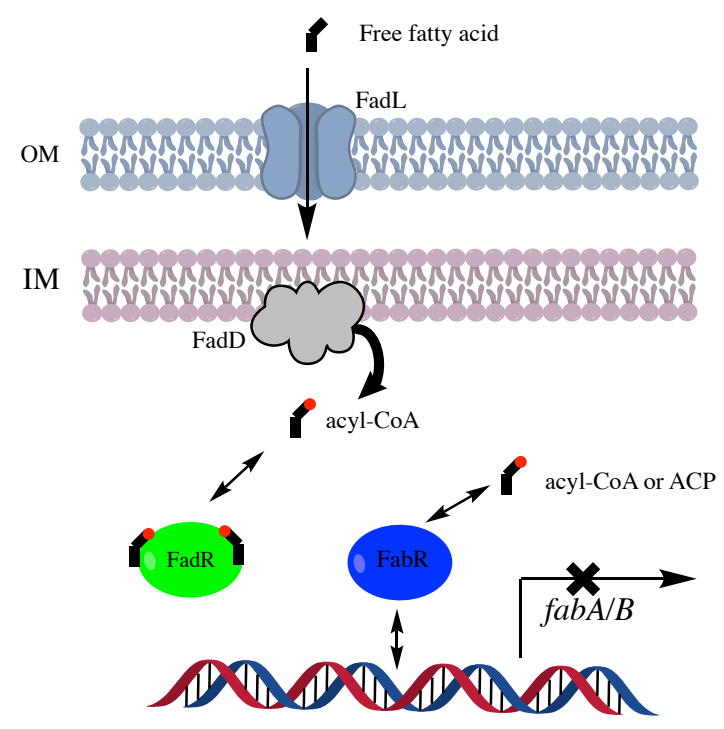




\section{Figure 2}

A

pfaA::lacZY

B

pfaA::lacZY
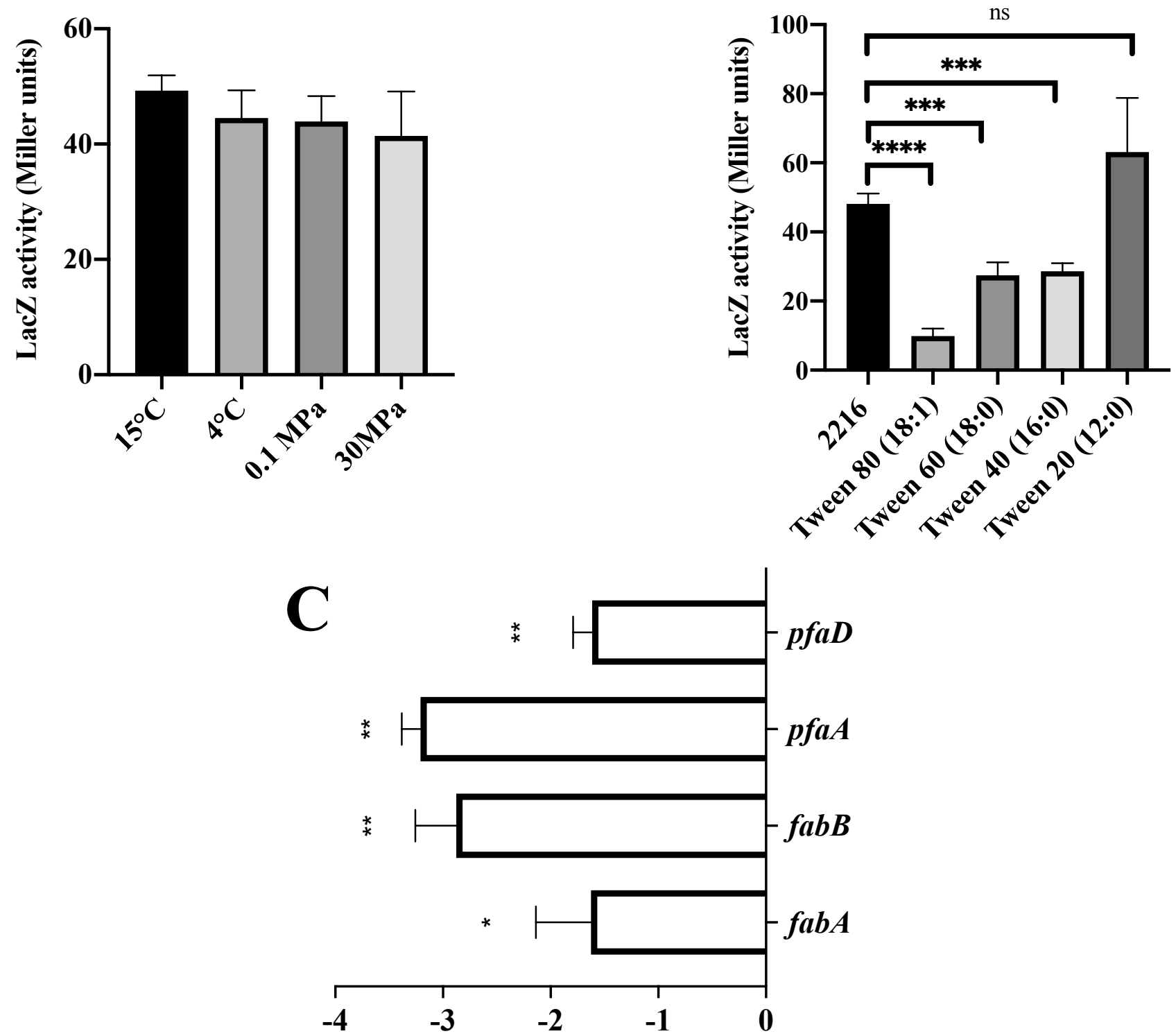

Relative mRNA abundance 


\section{Figure 3}
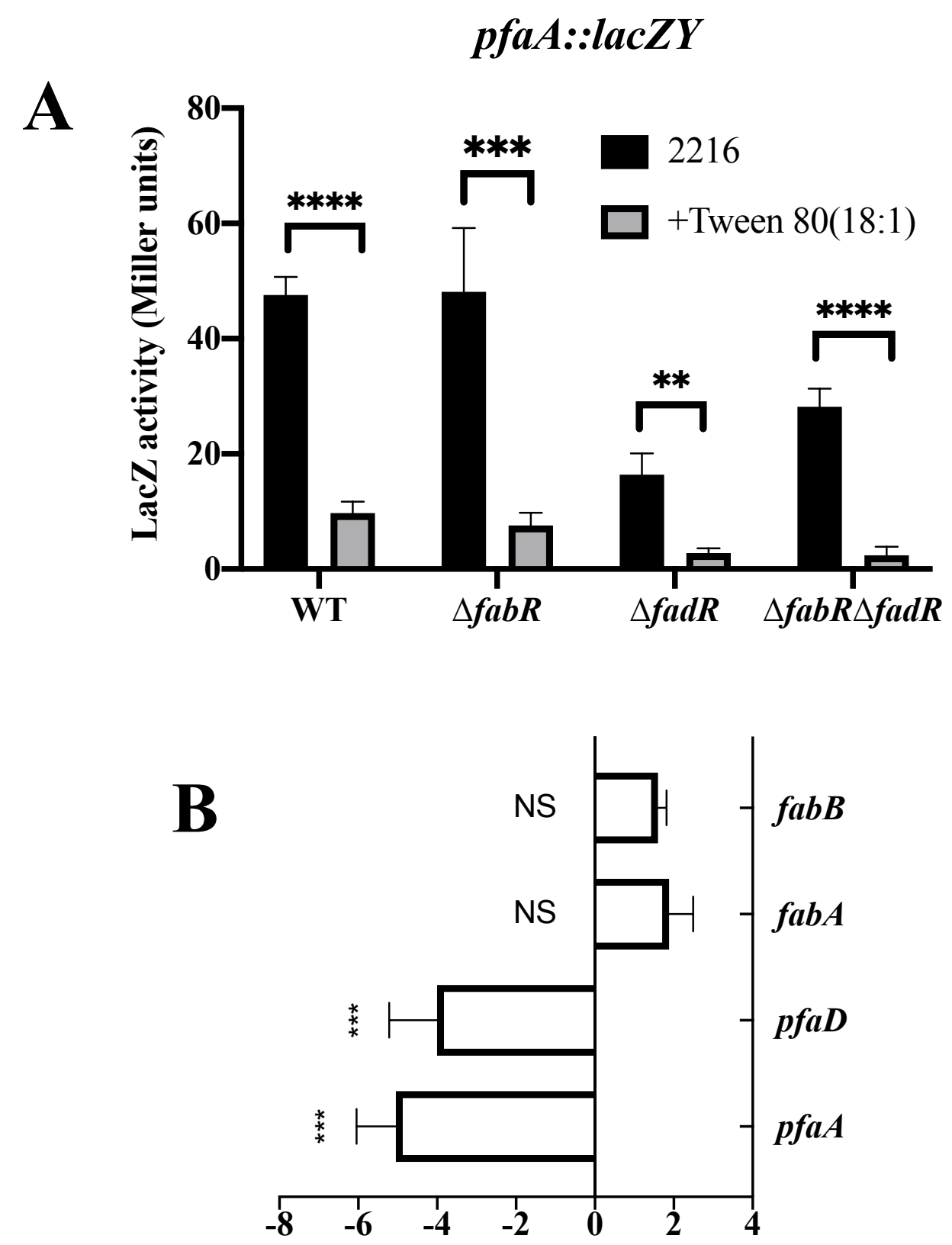

Relative mRNA abundance 


\section{Figure 4}

A

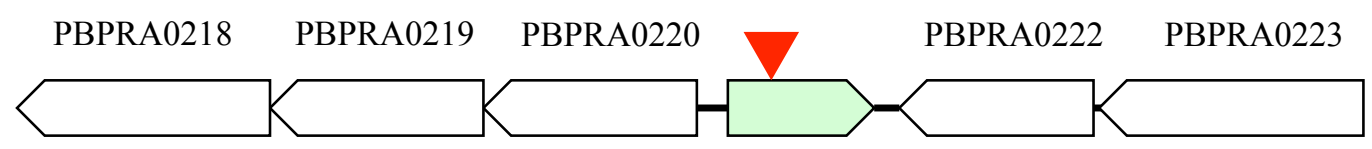

$\begin{array}{lccc}\text { Locus tag } & \text { Pfam hit } & \text { Function } & \text { E-value } \\ \text { PBPRA0218 } & \text { PF04932 } & \text { O-antigen ligase } & 3.2 \mathrm{e}-18 \\ \text { PBPRA0219 } & \text { Pfam03279 } & \text { Lipid A acytransferase } & 9.3 \mathrm{e}-81 \\ \text { PBPRA0220 } & \text { Pfam01370 } & \text { NAD dependent epimerase/dehydratase family } & 1.5 \mathrm{e}-42 \\ \text { PBPRA0221 } & \text { Pfam13972 } & \text { Bacterial transcriptional repressor } & 3.03 \mathrm{e}-38 \\ \text { PBPRA0222 } & \text { Pfam04748 } & \text { Divergent polysaccharide deacetylase } & 2.6 \mathrm{e}-72 \\ \text { PBPRA0223 } & \text { Pfam01551 } & \text { Peptidase family M23 } & 2.6 \mathrm{e}-21\end{array}$

B

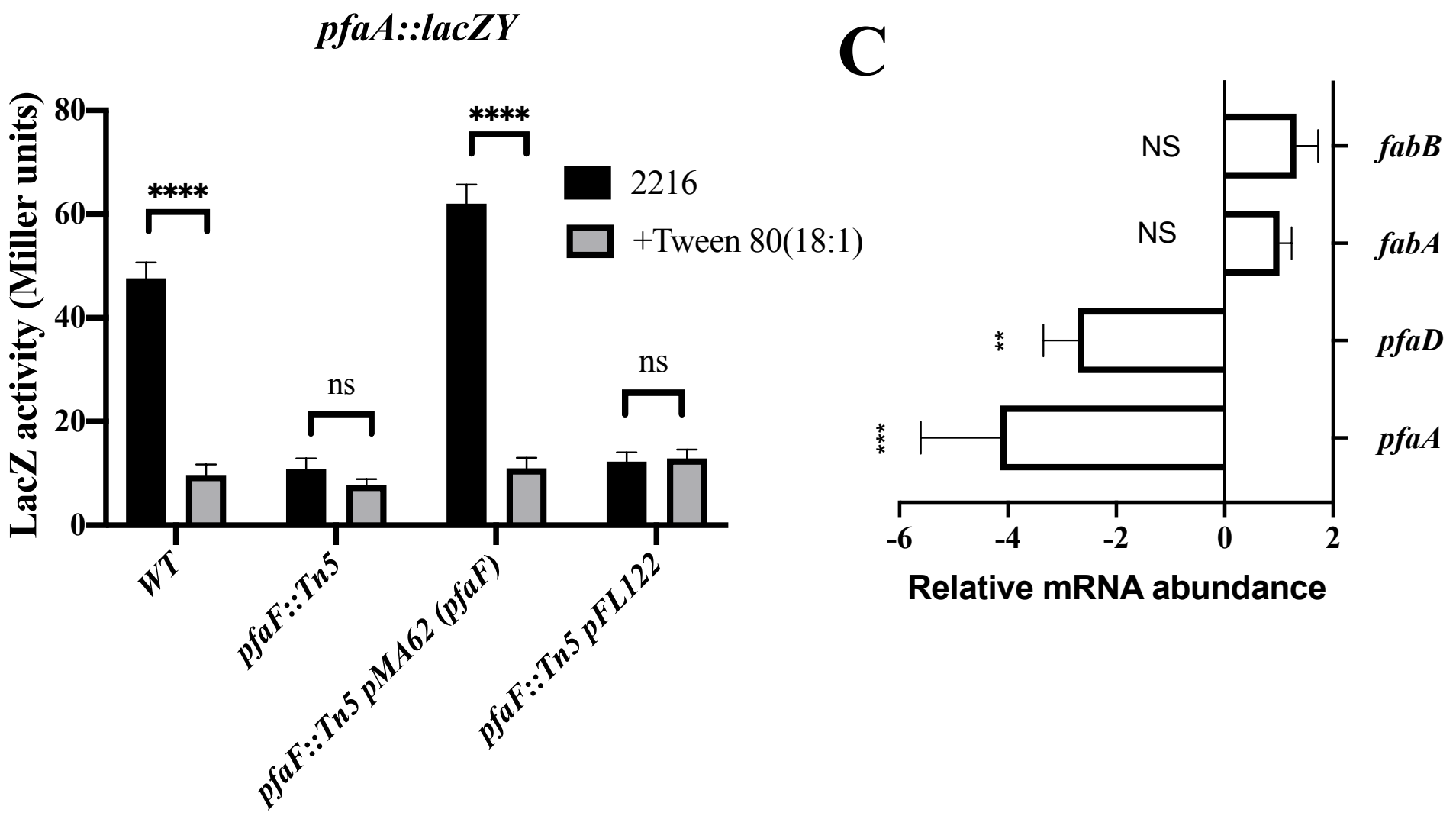


bioRxiv preprint doi: https://doi.org/10.1101/2020.01.28.924217; this version posted January 29, 2020. The copyright holder for this preprint (which was not certified by peer review) is the author/funder. All rights reserved. No reuse allowed without permission.

\section{Figure 5}

A

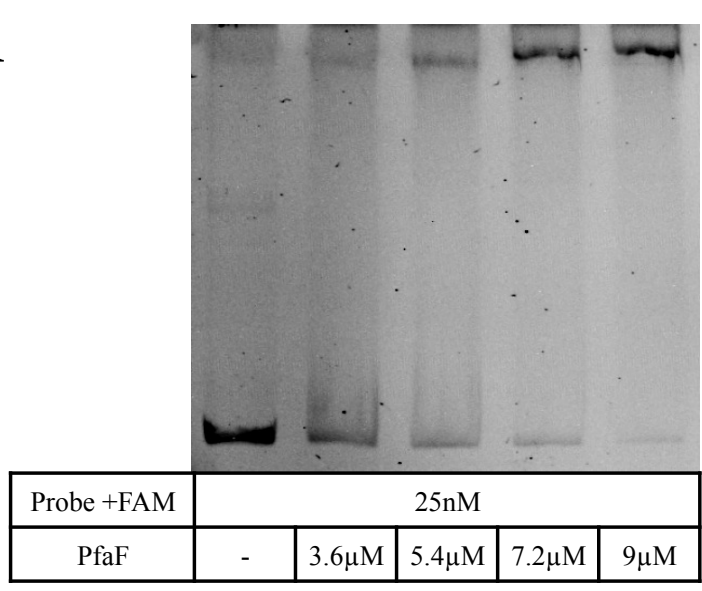

C

$\mathbf{E}$
B
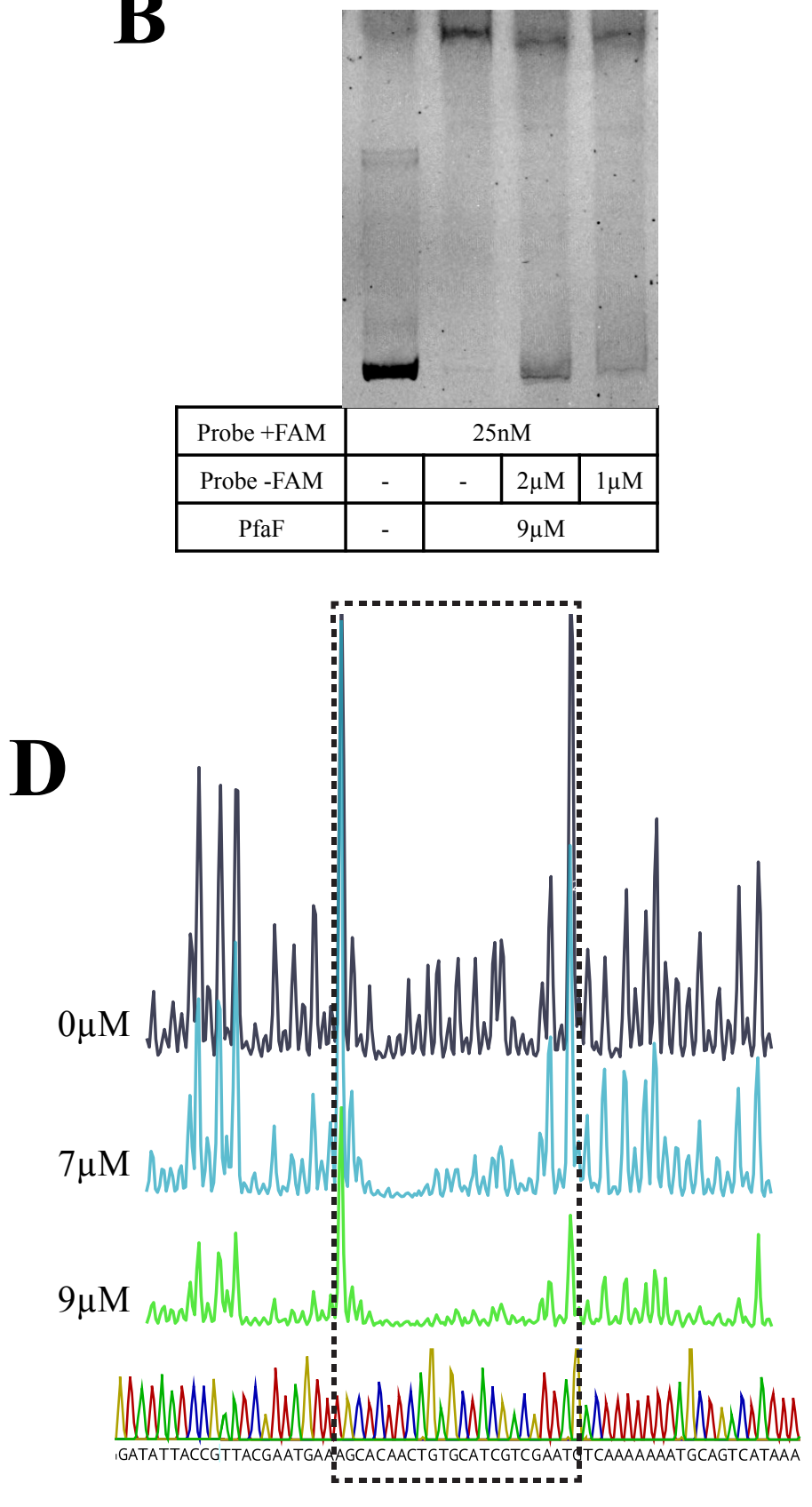

aactggtcttaagtgatccaacctcttgctccagtgattgtaacgaaaagcgtcctcgggtagtctgctcaac

cattaactgattaagcatgctttcattagggatggtaagtgcctccttatttgataatcgcagcagtagtaca

gcaggattatgatggatattacCgttacgaatgaaAGCACAACTGTGCATCGTCGA $\overline{\text { Atgtca }}$ aaaaatgcag tcataaatatgcataatctttctactcgtacattccgaagccatgacgctgccaatcaatttttagcgcctgt actcacacatgcgttatgccattttttcactcttatttttcaccagttgcctaatcgcaacgcccacaccgaa 


\section{Figure 6}

\section{$\underline{\text { Absence of exogenous fatty acids }}$}

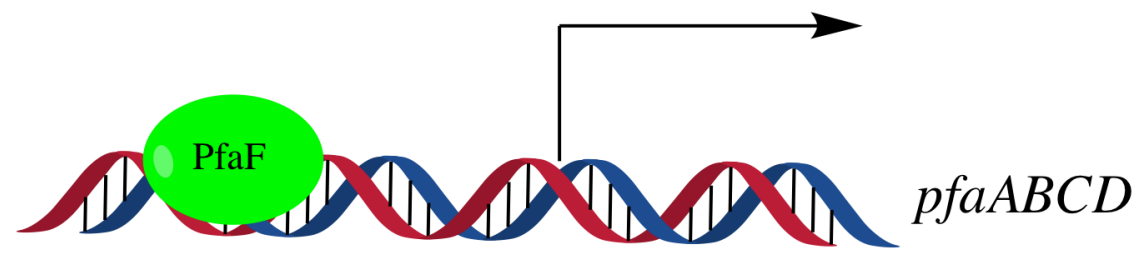

\section{Presence of exogenous fatty acids}

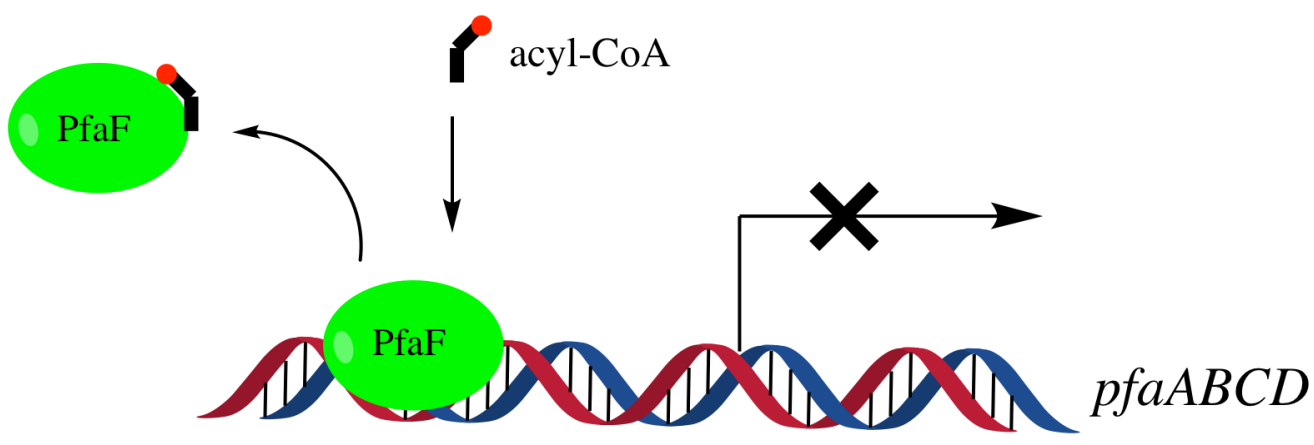

